\title{
Rain Attenuation Predicted Model for 5G Communication in Tropical Regions
}

\author{
Trilochan Patra, Swarup Kumar Mitra
}

\begin{abstract}
The signals operating at higher microwave frequency ranges get attenuated in the tropical regions where heavy rainfall occurs. Controlling of Signal fading for establishment of efficient link plays an important role in the heavy rainfall regions. Here rain attenuation predicted model has been designed in sub- $6 \mathrm{GHz}$ and mm Wave bands. This predicted model is applicable to the tropical regions where heave rainfall occurs. Frequency variation technique has been adopted to execute the research work. The estimated rain attenuation depends on International Telecommunication Union$R$ rain mitigation forecast technique utilizing assessment of rain in the tropical regions of South East Asia.The frequency ranges used here for variation techniques are respectively 3.6 to $4.2 \mathrm{GHz}, 4.4$ to $4.9 \mathrm{GHz}, 27.5$ to29.5 GHz, 37 to-40 GHz and 64 to71 GHz. In the previous works [1] it is observed that only lower fade margin has been considered for communication link design. As the fade margin increases, the communication link seems to be more reliable. In this paper the fade margin has been increased and it has been chosen from $12 \mathrm{~dB}$ to $16 \mathrm{~dB}$. This predicted model will yield better result than that of ITU-R model.
\end{abstract}

Keywords: Frequency variation technique, Frequency variation correction factor, Fade margin, Fade mitigation Technique.

\section{INTRODUCTION}

The process of dispatching a word from one side and receiving it on the other side between a certain distance without any wire and vice versa appeared in 1970s and this process started to put its footprint from $1 \mathrm{G}$ to 5G. Fifth generation technology requires very high data rate, higher system capacity, energy saving, cost reduction and higher bandwidth, which have not been used before. The 5G technology requires various kinds of new advanced features which have made it most powerful for the future.

A predicted model for $5 \mathrm{G}$ communication has been designed in this paper. This designed predicted model is very inevitable in the tropical countries or sub-tropical regions. With the help of this predicted model the signal attenuation due to heavy rainfall can be minimize frequency diversity technique. In the

Revised Manuscript Received on February 10, 2020.

* Correspondence Author

Trilochan Patra, Department of ECE, Techno International Newtown, Rajarhat, kol- 700156, India. E-mail trilochanpatra266@gmail.com.

Swarup Kumar Mitra, Department of ECE, MCKV Institute of Engineering,Liluah,Howrah-711204, India E-mail swarup.subha@gmail.com

(C) The Authors. Published by Blue Eyes Intelligence Engineering and Sciences Publication (BEIESP). This is an open access article under the CC BY-NC-ND license (http://creativecommons.org/licenses/by-nc-nd/4.0/) paper [1] the fade margin has been Considered up to $7 \mathrm{~dB}$. With the increment of fade margin the communication link becomes more reliable. In this paper the fade margin has been taken up to $16 \mathrm{~dB}$ to enhance the reliability of the communication link. With the increment of

frequency and due to heavy rainfall the signal gets attenuated which requires high static fade margin [2]. In this paper the Static fade margin has been enhanced to make it applicable for higher microwave frequency regions.

In paper [4] frequency ranges is available up to $38 \mathrm{GHz}$. But in this paper the frequency ranges have been improved up to $71 \mathrm{GHz}$. This predicted model is applicable to the higher frequency regions where higher fade margin prevails. When microwave radio frequency signal is scattered and absorbed by rain, signal gets attenuated [7]. In the tropical regions the signal power level is diminished on account of high rain rate and as a result rain fade occurs and this phenomenon leads to poor quality communication system which seems to be unreliable. In the tropical areas having high rain intensity the signal is faded and it causes a fatal problem for microwave link design. Now a day the Telecommunication technology requires a highly reliable system having both higher speed and increased frequency bands. Using diversity scheme, the authenticity and attributes of communication can be developed, which has been accepted as a very powerful process for rain attenuation to be diminished. In the frequency diversity technique, the signal is transmitted using different frequency channels affected by frequency selective fading [1]. The diversity scheme provides a method to develop the dependency of dispatched information utilizing two or more transmission avenues containing various characteristics. Henceforth the percentage of time of link failure is diminished.

A divination design of microwave connection is required to analyze the effect of rain rate. A prediction model has been proposed by ITU for estimation of the rain attenuation level. Among different rain mitigation predicted patterns ITU-R shape is mostly used form [13]. Here the rain mitigation predicted model has been considered for 5G communication and this model is expected to give the better result than ITU-R model.

In the heavy rainfall regions signal gets attenuated and this signal attenuation can be minimized using fade mitigation technique which is indicated in section II. Different rain attenuation predicted models have been defined in section III. In the same way the proposed methodology and the result analysis of the model have been described respectively under sections IV \& V. SectionVI indicates only conclusion part of this paper.

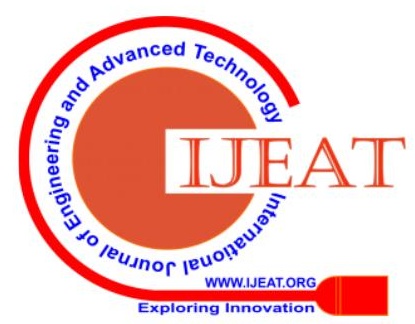




\section{Rain Attenuation Predicted Model for 5G Communication in Tropical Regions}

\section{FADE MITIGATION TECHNIQUE}

In the tropical regions a signal gets attenuated due to heavy rainfall and this signal attenuation can be minimized using Fade Mitigation Technique (FMT). To minimize rain fade there exist also different diversity approaches like diversity of time, diversity of space, diversity of frequency etc. The tropical regions also belong to another two types of fade mitigation techniques like adaptive power control technique and adaptive transmission technique. In the adaptive power control technique, the signal quality is degraded on account of propagation effect. This signal degradation can be compensated by maintaining a wanted level of power at receiver side either increasing antenna gain or increasing carrier power at the terminal by varying the energetic isotropous emitted power. If link power is allowed to be developed, the availability of the link in the heavy rainfall regions can improve. By utilizing uplink power control or downlink power control a power can be adjusted called carrier power. The restriction of power by the uplink has been accepted as the simplest way of rain fade mitigation. [16].

The adaptive transmission process changes the way of signal processing and transmitting it into the communication link. This technique overcomes rain attenuation either by using encoding technique or modulation technique. This technique comprises data rate compression technique, adaptive modulation technique and adaptive coding technique. In the data rate compression technique, the bandwidth of the information signal is minimized as the data rate is matched with the level of propagation effect. As a result, the ratio between the signal power and noise power increases. Adaptive modulation process varies the modulation technique between two conditions where as one condition lies under clear air state where a modulation scheme of high spectral efficiency is used and the other lies along attenuated avenue in which poor efficiency but vigorous modulation strategy has been employed [2]. The compatible coding process has been used for error correction and apprehension. These errors originate from signal propagation effect i.e. forward error correction. FMT for the physical layer are briefly described below [16]-

\section{A. Power control Technique:}

There are four categories of power controlling fed mitigation concepts, up-link control of power, end-to-end control of power control, down-link control of power control, and size of on-board beam. The purpose of up-link control to control power is to match the power product from a communication earth station with uplink loss. The transmitter power is countered to fade when the diffusion condition is corrected enough to limit interference to the apparent diffusion. In the case of apparent payloads, ULPC can resist the reduction of the equivalent isotropically radiated power (EIRP) of the satellite, which is caused by the uplink power level decreasing in the absence of UPC. EEPC is used for a positive figure of concentration. For regenerative repeaters, none of the uplink and downlink budgets depend on others so that the concept of EEPC does not exist in any way.

\section{B. Adaptive waveform Technique:}

These FMTs can be divided into three phases. These are Adaptive Coding (AC) technique, Adaptive Modulation (AM) technique and Data Rate Reduction (DRR) technique. The introduction of plentiful bits into information bits, while experienced by a fading one link, allows detection and correction of errors created by propagation losses and this at the lack of energy required per information bit Causes to go. Adaptive coding techniques help to implement a variable coding rate that coincides with the loss arising from the propagation conditions. The higher method for a given bandwidth can be obtained from the spectrally efficient modulation scheme but only in clear sky conditions due to the limit budget. The mission of the adaptive modulation technique is to reduce the required energy per information bit according to the results of employment of a lower-level modulation technique. Further reduction can be achieved by reducing the information data rate at constant bar. The technique is named data rate reduction. Here the user data rates must be matched with the diffusion conditions. Nominal data rates are applied under clear sky conditions where there is no deterioration in service quality with respect to system margins and a decrease is added according to the fade level. ULPC on the other hand aims to restore the carrier to sound ratio by augmenting the transmitted power of the earth station. Coding and data rate reduction allow the required C / N0 to be reduced, while link performance is maintained over the duration of the BER. The purpose of AC and AM is to reduce the required energy-per-information bit level (Eb / N0) and to decrease the required C / N0.

\section{Mitigation Technique at Layer 2}

The fade mitigation technique at the layer 2 level is not aimed at reducing a fade phenomenon. Instead, the fade mitigation technique relies on re transmission of the message. Two different techniques are followed at layer 2: Automatic Repeat Request (ARQ) and Time Diversity (TD). With the help of ARQ technology, messages are transmitted repeatedly until the message successfully reaches the receiving segment. ARQ with random time iteration can serve as an alternative solution. The time variation can be thought of as an FMT intended to re transmit information while allowing the status of the propagation channel to be received through it. In this case, there is no need to get the data file in absolute time and it is acceptable from the user's point of view to end the propagation event (normally a few tens of minutes) or to reduce traffic. The benefits of this technique derived from the use of a medium-term prediction model of propagation are used to calculate the most appropriate time to re-broadcast the message without request.

\section{Variation Technique:}

These techniques aim to disseminate information from different paths in the network to avoid destruction due to atmospheric disturbances. Therefore three types of variation techniques can be chosen: site variation (SD), satellite variation (SatD) and frequency variation (FD). These techniques are very expensive because the equipment concerned is excessive. 
SD is installed on the change of network paths, so it is applicable only for fixed satellite service. SD has an advantage in that the two fades assembled by two Earth stations separated by a certain distance (at least $10 \mathrm{~km}$ ) are greater than the size of a convective rain cell and are statistically independent. An earth station affected by a weak event is used and its information is sent to the original terminus via a separate terrestrial network. The current paper is used in the frequency variation technique because in this technique the signal is spread over a large bandwidth. The frequency variation technique and frequency variation correction factor are briefly discussed below-

- $\quad$ Frequency Variation Technique:

The frequency variation technique is applicable for the transmission of the same message signal at different carrier frequencies. To obtain received signals that are statistically independent or at least uncorrelated, the difference between the carrier frequencies will exceed the radio channel's coherence bandwidth. Coherence bandwidth depends on the multipath delay spread over the channel. One limitation of this technique is the inability of the receiver to detect all signals i.e. this technique requires multiple receivers at the receiver site. This is not really meant to replicate the message signal at two different frequencies, as it reduces spectral efficiency. Instead, the signal is spread over a large bandwidth, so parts of the signal are transmitted by different frequency components. Such propagation of signals can be done in various ways. These methods include multicarrier modulation using inverse DFT and serving frequency for widely varying frequencies from burst to burst. The signal is transmitted using multiple frequency channels. Frequency diversity achieves a single transmit / antenna pair, but operates at two or more widely spaced carrier frequencies and is shown in Figure 1

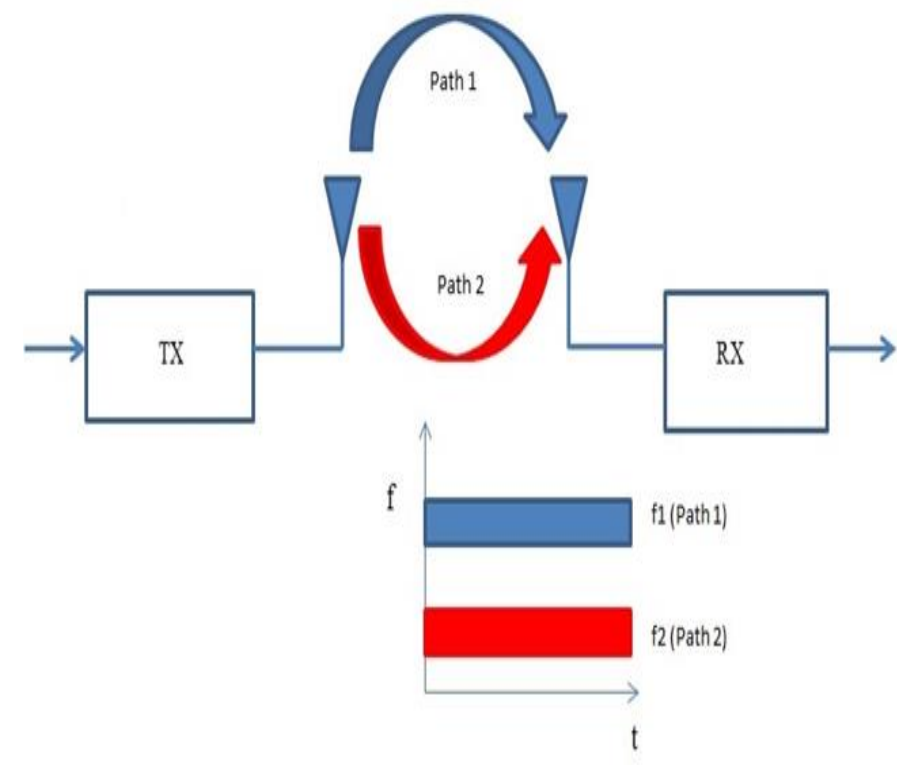

Fig.1. A generic user centric 5G communication

\section{- Frequency Variation correction Factor}

The performance of frequency variation can be described as an outage percentage of time [16]. The diversity improvement factor or variation correction factor (I) is described as the ratio between the outage percentage of an exact fade margin with no variation $\mathrm{P}_{\mathrm{ND}}(\mathrm{A})$ and the outage percentage with the same fade margin in the variation frequency $\mathrm{P}_{\mathrm{WD}}(\mathrm{A})$.

$\mathbf{I}=\mathbf{P}_{\text {ND }}(\mathbf{A}) / \mathbf{P}_{\text {WD }}$ (A)

As the definition developed in Equation (1) depends on the desired attenuation, frequency deflection and outage level at base frequency or variation frequency, specific attenuation of rain in terms of frequency is considered as a very important event in terms of progress or correction factor.

\section{DIFFERENT RAIN ATTENUATION MODELS}

A large number of systems have been designed by many researchers to overcome rain attenuation, especially at high frequencies. Various rainfall attenuation predictive models developed from time to time and the International Telecommunications Union Radio Communications Sector (ITU - R) model [6] are highly accepted throughout the world for rainfall attenuation estimation and therefore a comparison with the reliability of developing models with developed models is inevitable and especially for cases where measured data is not available [17]. There are various models for rainfall estimation forecast, but the following models are considered according to performance, namely, ITU-R, SAM, GarciaLopez [18], Karsawa, Maufouma and Crane Global model [18]. These models are chosen for their reliability, accuracy, and extensive survey conducted previously. Table I gives a comparison of the above precipitation attenuation model-

Table-I: Comparison among different existing predicted models

\begin{tabular}{|l|l|}
\hline \multicolumn{1}{|c|}{ Models Name } & \multicolumn{1}{c|}{ Limitation } \\
\hline ITU-R & It is good only for low attenuation \\
\hline Garcia-Lopez & This model is accurate only for Northern India \\
\hline SAM & $\begin{array}{l}\text { This model is simple but inaccurate for } \\
30 \mathrm{~mm} / \mathrm{hr} .\end{array}$ \\
\hline Karasawa & Applicable for Japanese environment. \\
\hline Moupfouma & $\begin{array}{l}\text { This model gives the highest attenuation for a } \\
\text { low frequency of 10 GHz }\end{array}$ \\
\hline Crane Global & $\begin{array}{l}\text { Gives high attenuation forecast for low } \\
\text { rainfall areas }\end{array}$ \\
\hline
\end{tabular}

\section{IV.PROPOSED METHODOLOGY}

By applying frequency variation technique the new rain attenuation predicted model has been designed that can yield the better result than ITU-R model. Hence the proposed methodology for designing the rain attenuation predicted model has been divided into three parts. 


\section{Rain Attenuation Predicted Model for 5G Communication in Tropical Regions}

These are block diagram; flow chart and algorithm for proposed model have been depicted in subsection A, B and C respectively.

\section{A.Block Diagram}

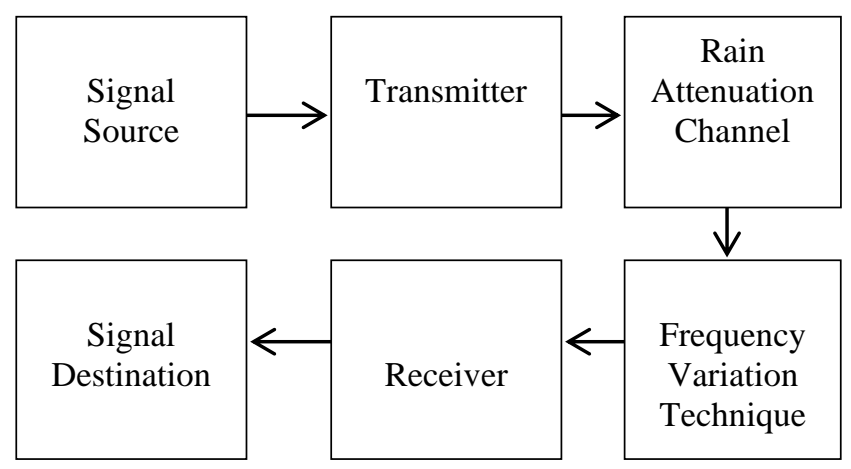

B. Flow Chart

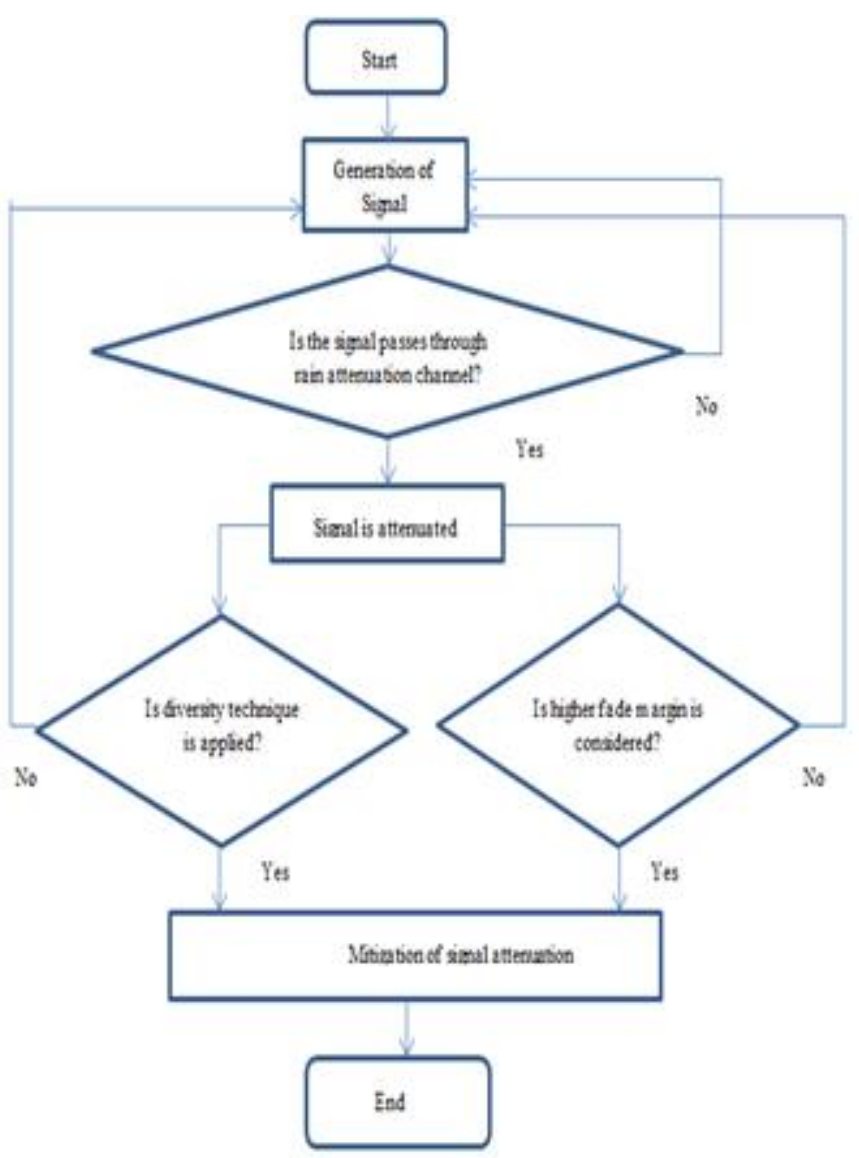

\section{Algorithm for proposed model}

The model proposed in this work is applicable to high rainfall attenuation; high frequency and high fade margins. It is also suitable for all natural conditions and areas with high rainfall. For high rainfall rates the probability of gamma distribution applies [19] which can be expressed for $0.01 \%$ outage in the following way

$\Gamma\left(10^{-4}\right)=\int_{0}^{\infty} s^{10^{-4}-1} e^{-s} \mathrm{ds}, 10^{-4}>0$
Where $\Gamma\left(10^{-4}\right)$ is the gamma function, $\mathrm{S}$ is a function. The gamma distribution is used to represent high rainfall rates because the total percentage of precipitation is normally of the order of $2 \%$ to $10 \%$. The proposed model is designed for a frequency range of $3.6-4.2 \mathrm{GHz}, 4.4-4.9 \mathrm{GHz}$. , Is 27.5-295 GHz, 37-40 GHz, and 64-71 GHz. The length of the microwave path is $1 \mathrm{~km}$ and the fade margin is developed from $12 \mathrm{~dB}$ to $16 \mathrm{~dB}$. Now the ITU-R model [5] [14] for specific rainfall attenuation is represented by the following equation (3) -

$$
\gamma=\mathbf{k} \text { R\% Po dB / km }
$$

Where $\mathrm{k}$ and $\alpha$ denote the scattered co-efficient. $\mathrm{R} \% \mathrm{P}$ denotes the rate of rain outside the $\mathrm{P}$ percentage. $\mathrm{P}$ is the percentage time of rainfall attenuation that exceeds the margin limit. The values of $\mathrm{K}$ and $\alpha$ are frequency dependent. The values of $\mathrm{k}$ and $\alpha$ for the above frequency ranges can be calculated from the following equations [20] -

$$
\begin{aligned}
& \log k=\sum_{m=1}^{3}\left[a_{m} \exp \left\{-\left(\frac{\log f-b_{m}}{c_{m}}\right)^{2}\right\}\right]+m_{k} \log f+c_{k} \\
& \alpha=\sum_{l=1}^{4}\left[a_{l} \exp \left\{-\left(\frac{\log f-b_{l}}{c_{l}}\right)^{2}\right\}\right]+m_{\alpha} \log f+c_{\alpha}
\end{aligned}
$$

Where $\mathrm{f}$ is the base and diversity frequency.

$\mathrm{k}$ : Either $\mathrm{k}_{\mathrm{H}}$ for horizontal polarization or $\mathrm{k}_{\mathrm{v}}$ for vertical polarization.

$\alpha$ : Either $\propto_{\mathrm{H}}$ for horizontal polarization or $\alpha_{\mathrm{V}}$ for vertical polarization).

$a_{m}, b_{m}, c_{m}, a_{l}, b_{l}, c_{l}, m_{k}, c_{k}, m_{\alpha}, c_{\alpha}$ are the co-efficient.

Table- II: Values of $k$ and $\alpha$ for aforesaid frequency ranges.

\begin{tabular}{|c|c|c|}
\hline $\begin{array}{c}\text { Frequency } \\
\text { (GHz) }\end{array}$ & $\boldsymbol{k}_{\boldsymbol{H}}$ & $\boldsymbol{\propto}_{\boldsymbol{H}}$ \\
\hline 3.6 & 0.000516 & 0.9582 \\
\hline 4.2 & 0.000723 & 1.14498 \\
\hline 4.4 & 0.000768 & 1.1688 \\
\hline 4.9 & 0.001046 & 1.2275 \\
\hline 27.5 & 0.1545 & 1.0308 \\
\hline 29.5 & 0.1667 & 1.0198 \\
\hline 37 & 0.2968 & 0.9628 \\
\hline 40 & 0.3504 & 0.9334 \\
\hline 64 & 0.7673 & 0.8253 \\
\hline 71 & 0.8635 & 0.7939 \\
\hline
\end{tabular}

The measured rainfall rate in southeast Asia is $118 \mathrm{~mm} / \mathrm{hr}$ for predicting specific rainfall attenuation for the above frequency. Has been used. Using equation (3) at $0.01 \%$ for the above frequency ranges and measuring the rate of rain the following graphs are plotted for specific rainfall attenuation vs. outage percentage in Southeast Asia: -

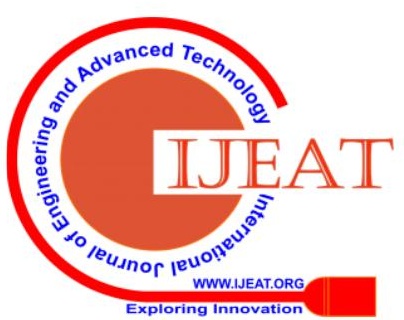




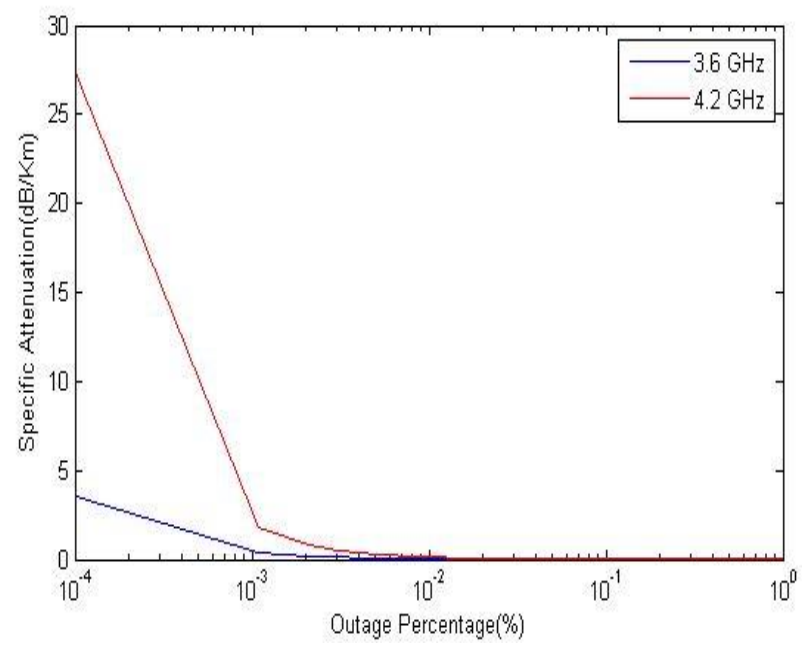

Fig 2: For 3.6-4.2 GHz

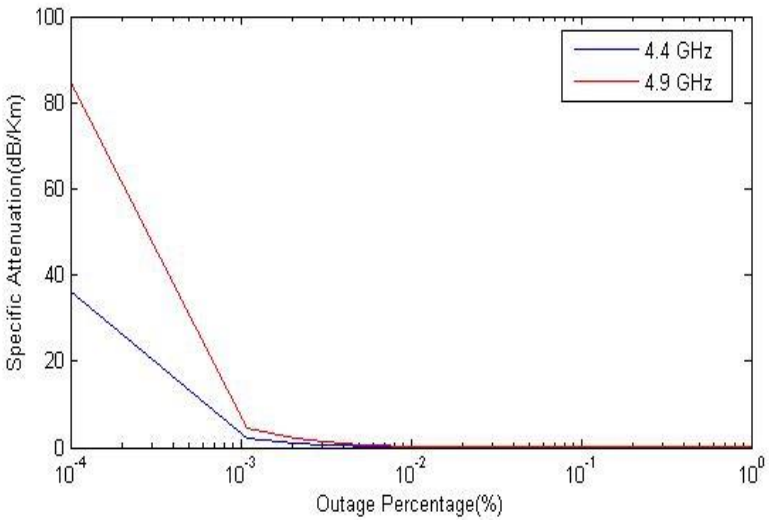

Fig.3.For 4.4-4.9 GHz

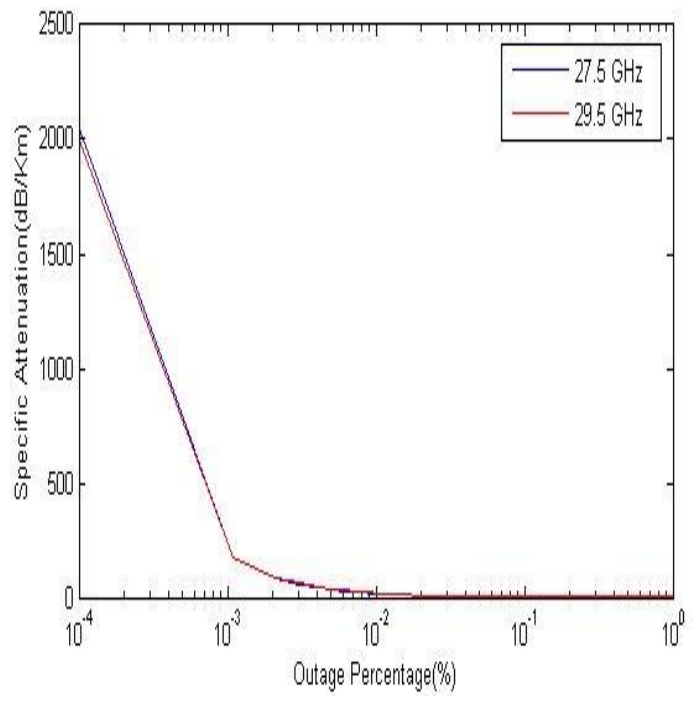

Fig.4.For 27.5-29.5

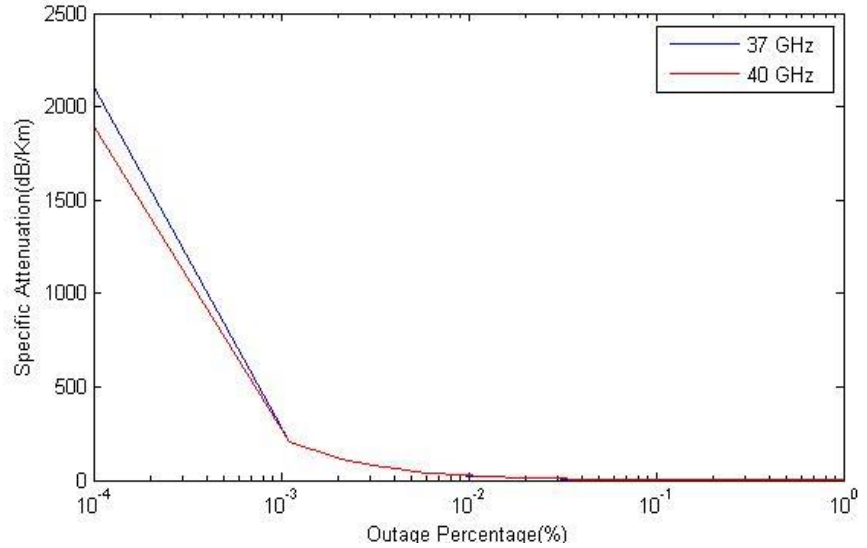

Fig.5. For 37-40 GHz

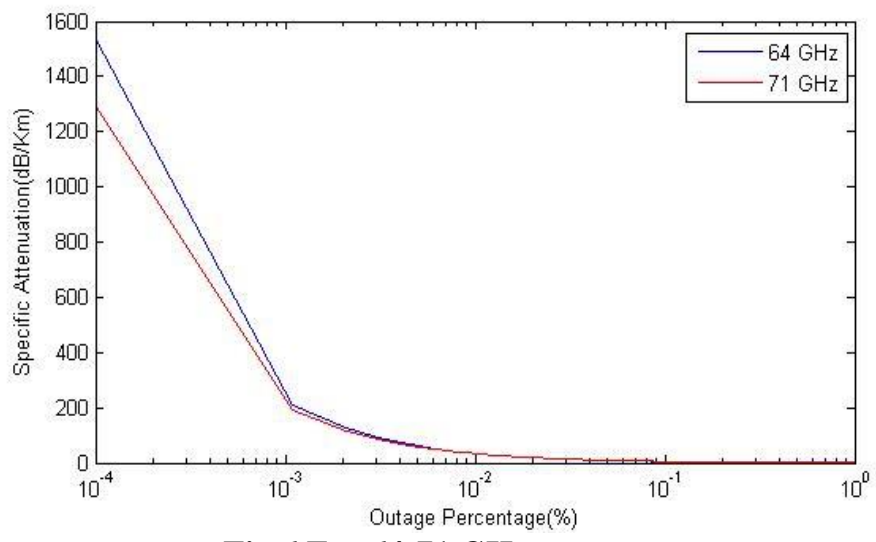

Fig.6.For 64-71 GHz

The above figures (Fig2, 3, 4, 5 and 6) show the change of outage percentage with respect to specific rain attenuation for different fade margins required for the system. The fade margin for the system is chosen from $12 \mathrm{~dB}$ to $16 \mathrm{~dB}$. The above equation (1) for the above frequency ranges and fade margins applies to the above results as shown in Fig. 2, 3, 4, 5 and 6 . The estimated correction factors are shown in Table III below [7] [16]-

Table- III: Correction Factor Table (1) from the definition defined in the above equation

\begin{tabular}{|c|c|c|c|c|c|c|}
\hline \multirow{2}{*}{$\begin{array}{c}\text { Diversity } \\
\text { frequency } \\
\left(\mathbf{f}_{\mathbf{d}}\right)(\mathbf{G H z})\end{array}$} & \multirow{2}{*}{$\begin{array}{c}\text { Base } \\
\text { frequency } \\
\left(\mathbf{f}_{\mathbf{b}}\right)(\mathbf{G H z})\end{array}$} & \multicolumn{5}{|c|}{ Improvement Factor or correction Factor } \\
\cline { 3 - 7 } & & $\mathbf{1 2} \mathbf{~ d B}$ & $\begin{array}{c}\mathbf{1 3} \\
\mathbf{d B}\end{array}$ & $\mathbf{1 4} \mathbf{~ d B}$ & $\begin{array}{c}\mathbf{1 5} \\
\mathbf{d B}\end{array}$ & $\begin{array}{c}\mathbf{1 6} \\
\mathbf{d B}\end{array}$ \\
\hline 3.6 & 4.2 & 1.66 & 2.5 & 4.09 & 5.0 & 7.8 \\
\hline 4.4 & 4.9 & 1.25 & 1.6 & 1.83 & 2.7 & 3.33 \\
\hline 27.5 & 29.5 & 1.0 & 1.03 & 1.06 & 1.11 & 1.15 \\
\hline 37 & 40 & 1.2 & 1.36 & 1.25 & 1.33 & 1.4 \\
\hline 64 & 71 & 1.0 & 1.01 & 1.02 & 1.2 & 1.26 \\
\hline
\end{tabular}




\section{Rain Attenuation Predicted Model for 5G Communication in Tropical Regions}

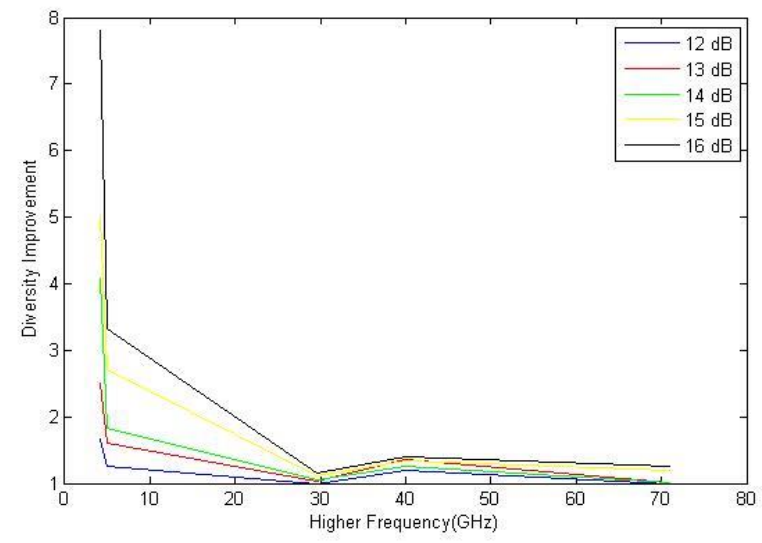

Fig.7. Correction factor for aforesaid frequency ranges in frequency variation scheme

In order to develop an approximate model, each graph in fig 7 represents an equation between the correction factor and the variance frequency. To obtain a model by combining these equations, all curves are fitted with respect to the fade margin (F) as shown in Fig. 8.

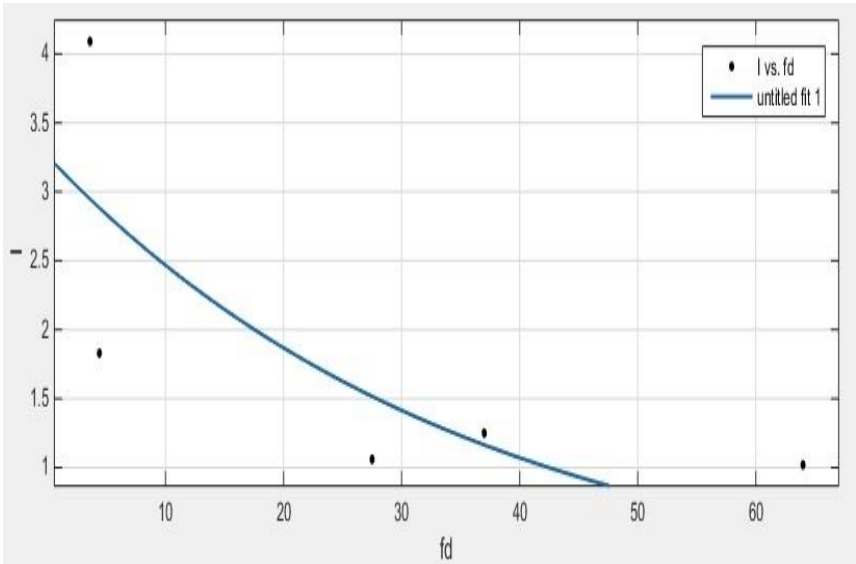

Fig.8. Approximate correction factor fitting at $14 \mathrm{~dB} / \mathrm{km}$ fade margin

Fig. 8 illustrates the general frequency variation correction model as best fit by equation (6) below.

$\mathbf{I}=\mathbf{a e} \wedge(\mathbf{b f d})$

(6)

Where A and B are coefficients related to fading and each has a value for different fade margins that is predicted and represented in Table 4 [7] [16] and $f_{d}$ is the variation frequency. The coefficient a, $\mathrm{b}$ is also depicted in figure 9 .

Table- IV: Model co-efficient values

\begin{tabular}{|c|c|c|}
\hline $\mathbf{F ( d B / K m ) ~}$ & $\mathbf{a}$ & $\mathbf{b}$ \\
\hline 12 & 1.456 & -0.006848 \\
\hline 13 & 2.106 & -0.01438 \\
\hline 14 & 3.258 & -0.02782 \\
\hline 15 & 4.376 & -0.0349 \\
\hline 16 & 6.899 & -0.05235 \\
\hline
\end{tabular}

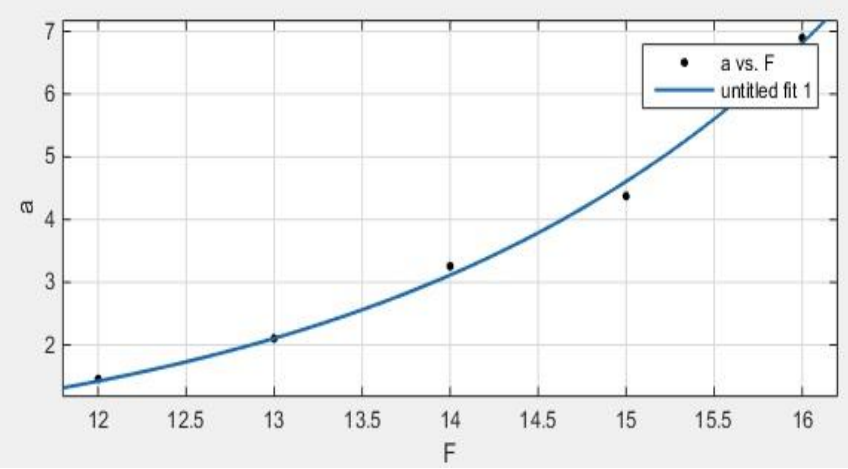

(a)

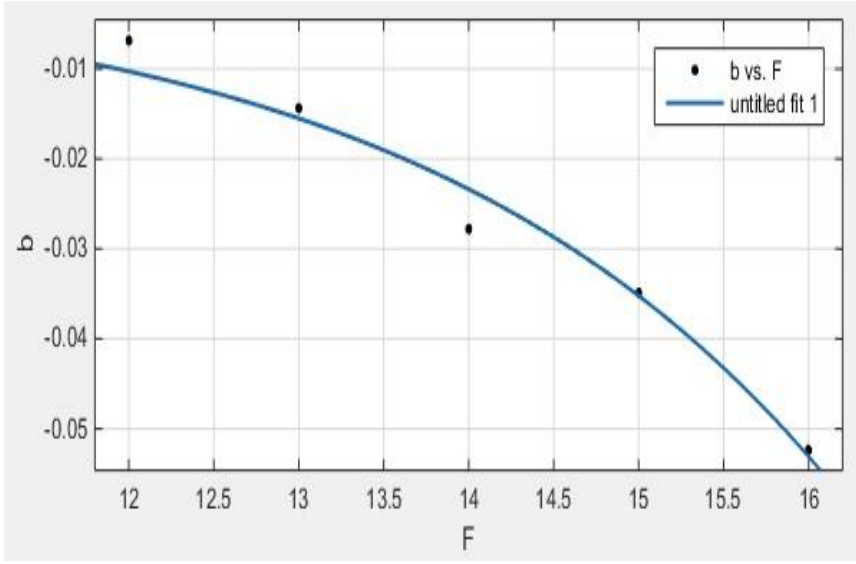

(b)

Fig.9. Plot (a) for co-efficient a and plot (b) for co-efficient b

\section{RESULT ANALYSIS}

The correction factor model using Equation (6) is estimated for the same specific rain attenuation and is shown in Figure 10 below-

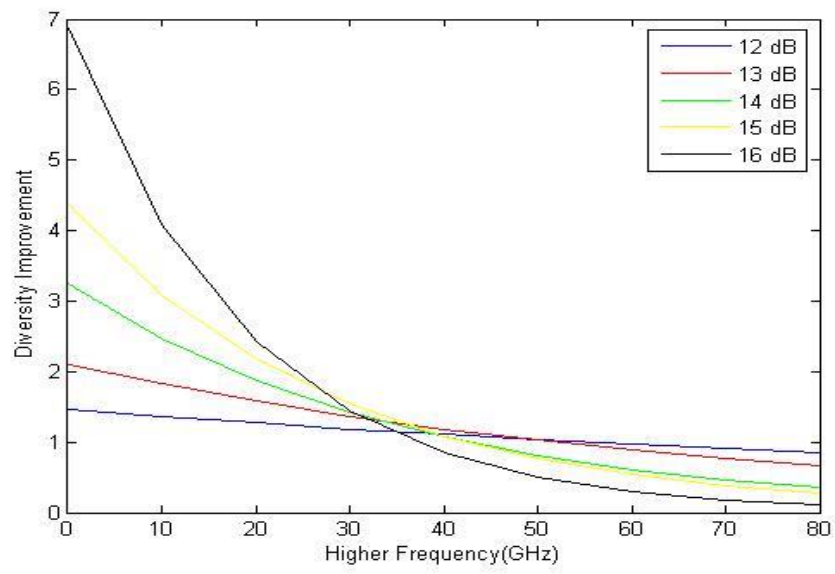

Fig.10. Correction factor dependent on proposed model

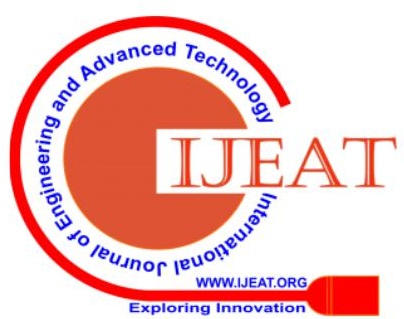


From figure 10 it is observed that as the fade margin increases the variation correction factor is minimal and for $16 \mathrm{~dB}$ fade margin the variation correction factor is minimum i.e. signal attenuation is minimum.

Comparison of fig 7 with fig 10 shows the difference between the diversity correction factor calculated by the general definition and the diversity correction factor predicted from the proposed model. Fig. 10 for the fixed fed margin calculates the reliability comparison for the improvement in the above frequency range based on the projected bulge frequency (estimated) and the ITU-R measured rain rate. From Fig. 11 the difference between the ITU-R model and the proposed model is very clear due to the proposed model being non-linear co-efficient.

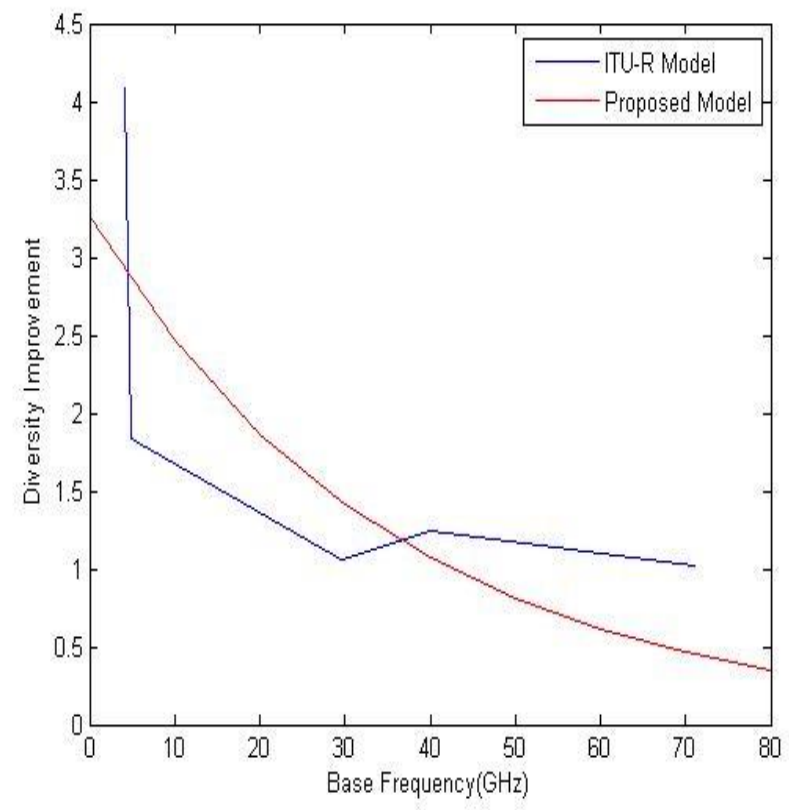

(a)

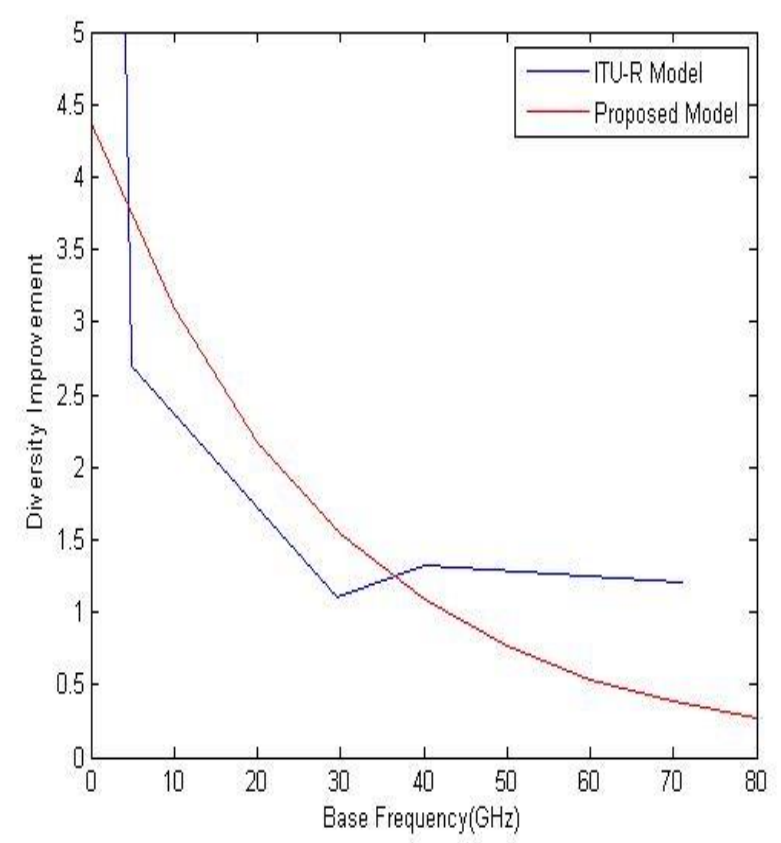

(b)

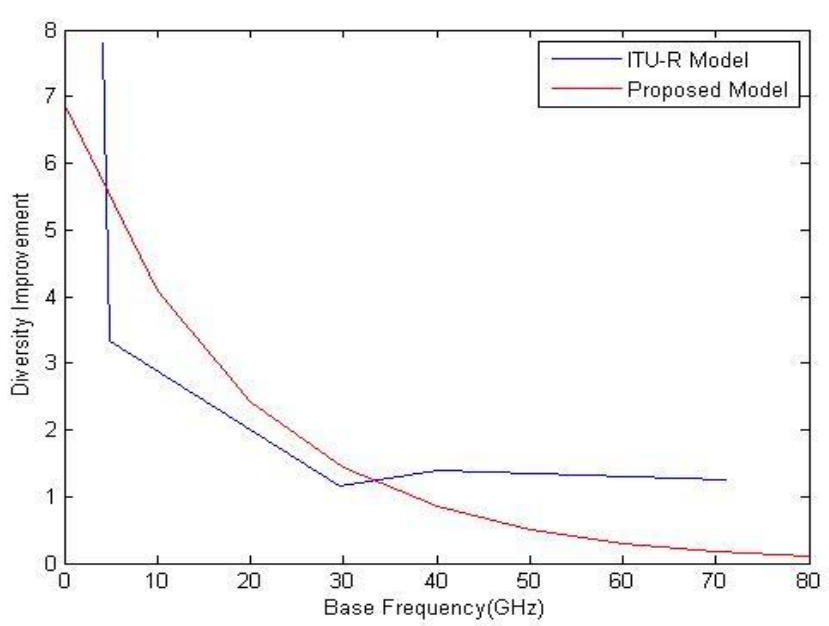

(c)

Fig.11. Predetermined and calculated frequency variation correction factor (A) $16 \mathrm{~dB} / \mathrm{km}$ (B) $15 \mathrm{~dB} / \mathrm{km}$ (C) $16 \mathrm{~dB}$ km From the above figures for the $14 \mathrm{~dB} / \mathrm{km}, 15 \mathrm{~dB} / \mathrm{km}$ and $16 \mathrm{~dB} / \mathrm{km}$ fade margins; it is observed that the variation improvement with respect to the base frequency is lower for the predictive model rather than the ITU-R model. We have considered the aforesaid frequency range for the above frequencies of 11 graphs of fig. We can also repeat this for other faded margins. So, the approximate model for the $5 \mathrm{G}$ communication frequency range gives better performance in the above fade margin. Reliability is also supported with rain attenuation measured from the $5-40 \mathrm{GHz}$ frequency range [1] and the 50-90 GHz frequency range [16].

\section{CONCLUSION}

Frequency variation can be applied using the proposed variation correction factor model for the above 5G frequency range based on an excessively faded margin for system designing. It is observed that the correction factor decreases for higher fade margins. For $5 \mathrm{G}$ communication i.e. for high frequency ranges when heavy rainfall occurs, rain attenuation for the $1 \mathrm{~km}$ path sometimes seems more important for reliability. It can be concluded that the hypothesized model provides better results than the ITU-R model. The proposed frequency diversity model is a very useful and important tool in the tropical regions in designing a reliable microwave communication link where the diversity or variation can be acquired according to the reliability of the system. This predicted model is very helpful to minimize the signal attenuation in the heavy rainfall regions. Besides it is applicable for higher fade margins so that the communication link will be more reliable and this predicted model will be easily accessible to all people of the tropical regions. 


\section{Rain Attenuation Predicted Model for 5G Communication in Tropical Regions}

\section{REFERENCES}

1. M. R. Islam, L.M. Altajjar, M.M. Rashid, and L.K. Bashar, International WIE Conference on Frequency Diversity Improvement Factor for Rain fed Mitigation in Malaysia, December 2015, pp.159-163 in Electrical and Computer Engineering, IEET, Dhaka, Bangladesh.

2. L. Castanet, a. B. Alamanek, and M. Bauset, Interference and fade mitigation techniques for $\mathrm{Q} / \mathrm{V}$ band satellite communications systems, ", Proc. Second International Workshop of COST Action, Vol. 280, January 2003.

3. B. D. Hodge, "An Improved Model for Diversity Gain on EarthSpace Propagation Paths', Radio Sci., Vol. 17, no.6, pp. 1393 1399, Nov.-Dec.1982.

4. M.R. Islam, J. Chebil and A. R. Tharek, "Frequency Scaling of Rain Attenuation From 23-GHz To 38-GHz Microwave Signals Measured in Malaysia", published in Asia Pacific Microwave Conference (APMC), IEEE, Singapore, Aug. 2002.

5. ITU-R "Propagation Data and Prediction Methods for Design of Terrestrial Line-of-Site Systems", ITU-R P.530-14, 2012.

6. ITU-R "Propagation Data and Prediction Methods Required for the Design of Earth-Space Telecommunication Systems", ITU-R P.618/11, 2013.

7. T. Patra, and S. Sil, Frequency Diversity Improvement Factor South-East Asia ', J. Using various MIMO techniques for rain fed mitigation in the mech. Ongoing. And mathematics. Sci., Vol.-13, No.-3, pp.87-102, July-August. 2018.

8. U. Kesavan, A.R. Tharek, s. K. a. Rahim, and M. R. Islam, "Review of rainfall attenuation studies in tropical and equatorial regions in Malaysia - an overview", Journal of Antennas and Propagation, IEEE, Vol. 55, No. 1, pp. 103-113, March 2013.

9. J. D. Lester, and W.W.L. Tutzman, "Frequency scaling of rain attenuation for satellite communication links", IEEE Transactions on Antennas and Propagation, Volume-43, Number 11, pp.12072016, Nov1995.

10. P. Majithiya, K. A. Sisodia, V. Muralidhar, and K. V. Garg, "Novel down link rain fade mitigation technique for Ka-band multi beam systems", International journal of Satellite communication and networking, Wiley Inter Science, Vol-25, no1, pp.45-51, Aug.2006.

11. IK Charilaos, and DP Athanasios, 'Ka Band and Above: Multiage Satellite MIMO Systems: Outage Capacity Analysis Using Bivariate IG Distribution', NEEE 8th European Conference on Antenna and Propagation (UAACAP), The Hague, Netherlands, April 2014, Pp. 6-11.

12. Q.W. Pan, J. E. Allnutt, and C. Tsui, “'Evaluation of Diversity and Power Control Techniques for Satellite Communication Systems in Tropical and Equatorial Rain Climates", IEEE Transactions on Antennas and Propagation, Vol. 56, no. 10, Oct.2008.

13. N. Sachdeva, D. Sharma, "Diversity: A Fading Reduction technique', International Journal of Advanced Research in Computer Science and Software Engineering (IJARCSSE), Vol. 2, no.6, pp-58-61, June 2012.

14. P. W. Raut, and S. L .Badjate, "Diversity Techniques for Wireless Communication', International Journal of Advanced Research in Engineering and Technology(IJARET), Vol. 4, no 2, pp. 144-160, Dec-2014.

15. Recommendation ITU-RP.530-16 (07/2015), "Propagation data and prediction methods required for the design of terrestrial line of sight systems," July 2015.

16. T. Patra, and S. Sil, "Frequency Diversity Improvement factor for Rain Fade Mitigation Technique for $50-90 \mathrm{GHz}$ in tropical region', IEEE conference (IEMECON), Thailand, August 2017, pp.86-90.

17. M. O. Fashuyi and T. J. Afullo, "Rain attenuation prediction and modeling for line-of-sight links on terrestrial paths in South Africa", Radio Sci., Vol. 42, no-5, Oct.2007.

18. Parth Panchal, Rutvij Joshi, "Performance Analysis and Simulation of Rain Attenuation Modelsat 12-40 GHz Band for an Earth Space Path over Indian Cities", 7thInternational Conference on Communication, Computing and virtualization, Dec-2016, pp.801-808.
19. ITU-R P.1057-1 "Probability distribution relevant to radio wave propagation modeling”, 2001.

20. ITU-R , P.838-2 “Specific attenuation model for rain for use in prediction methods, 2003

\section{AUTHORS PROFILE}

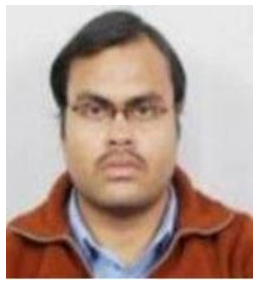

Trilochan Patra has obtained his B.Tech and M.Tech degree from MCKV Institute of Engineering under Maulana Abul Kalam Azad University of Technology, W.B India (Formerly known as West Bengal University of Technology) in 2009 and 2011. He is working as an assistant professor in ECE Department in Techno International Newtown (Formerly known as Techno India College of Technology) from 2011.His interested research area is Wireless communication, Microwave Communication etc.

E-mail:trilochanpatra266@gmail.com

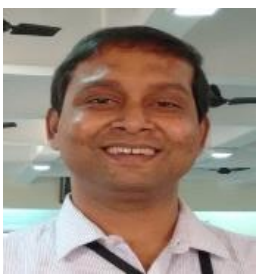

Swarup Kumar Mitra received his B. Tech degree from kalyani University, India in 2000. He has achieved his M.Tech in VLSI Design and Microelectronics technology from Jadavpur University, India in 2007. He is attached as associate professor in ECE in MCKV Institute of Engineering, Liluah, and Howrah, India and awarded with PhD (Eng.) 2012 from Jadavpur University. His present research area is wireless sensor network and its architecture etc.

E-mail:swarup.subha@gmail.com 